\title{
3-Dimensional Correlated Microscopy of Fluorescence Microscopy and Serial Block Face SEM
}

J. Mancuso ${ }^{1}$, J. L. Serrano-Velez ${ }^{2}$, I.I. Torres-Vazquez ${ }^{2}$, and E. Rosa-Molinar ${ }^{2}$

${ }^{1}$ Biological Imaging Group, University of Puerto Rico - Rio Piedras, San Juan, PR 00931

${ }^{2}$ Gatan Inc., Pleasanton, CA 94588

An essential biological question is "how is the three-dimensional geometry of cellular structures organized?" Researchers have two options for pursuing this question at the transmission electron microscope (TEM) level, tomography, and serial section imaging. Although tomography has excellent resolution, the field of view is limited and it is impractical for large volume acquisitions. Serial sectioning is used for obtaining large volume three-dimensional 3D electron microscopic data, but the technique is a highly skilled time consuming process prone to human error. Serial block-face scanning electron microscopy (SBFSEM) is a new automated technique in which a scanning electron microscope is used to obtain serial images. Bridging the gap between ultrahigh resolution tomography and fluorescence microscopy, SBFSEM provides a streamlined and automated 3D data acquisition process.

A microtome equipped with a diamond knife is mounted inside the chamber of the SEM and shaves off 50nm of the sample in between imaging. Images are collected using a back scatter (BS) electron detector which provides a classic TEM-like image. The SBFSEM imaging process is completely automated allowing for large volume acquisition in a timely manner without the risk of losing sections.

Adult Western Mosquitofish, Gambusia affinis (Gambusia hereafter) were anesthetized by immersion with benzocaine (1:2,000). Filter paper fibers saturated with fluorescent and/or non fluorescent gap junction-permeant and gap junction-impremeant tracers were implanted directly into crushed nerves innervating the deep muscles of the adult male gonopodium and of the adult female anal fin of Gambusia; fish were revived and the tracers was allowed to transport between 6.0-8.0 hrs. Gambusia were euthanized by immersion in benzocaine $(1: 4,000)$ and intracardially perfused with teleost buffer $\mathrm{pH} 7.4$ followed with $4 \%$ formaldehyde made from freshly depolymerized paraformardyde (PFA) in teleost buffer $\mathrm{pH}$ 7.4. After fixation, the spinal cord associated with vertebral segments 7-17 was removed, dissected free, and post-fixed overnight with $4 \%$ PFA in teleost buffer $\mathrm{pH}$ 7.4. Spinal cords were covered with mounting medium, cover-slipped and viewed using a Nikon C1 Laser Scanning Confocal Microscope. Three-dimensional (3D) volume reconstructions were rendered from light scanning confocal z stacks using the Imaris ${ }^{\circledR} 3 \mathrm{D}$ software. Volume reconstructions were obtained by thresholding intensity values and adjusting blending properties to create a solid and detailed volume model of the raw data acquired (see Figure 1A).

For SBFSEM, the coverslip was removed and the spinal cord was washed three times in teleost buffer $\mathrm{pH} 7.4$ to remove the mounting medium. The spinal cord was then post-fixed and stained with heavy-atoms. The spinal cord was dehydrated, infiltrated with resin, embedded and polymerized at $60^{\circ} \mathrm{C}$ for $48.0 \mathrm{hr}$. After polymerization, the resin-embedded spinal cord samples were serially shaved $(\sim 50 \mathrm{~nm})$, using a Gatan 3 View system. Serially imaging the block face rather than imaging individual sections, results in excellent registration between all images within an image stack (see Figure 1A). The labeling combined with the heavy atom staining provides high contrast at neuronal boundaries, in order to easily contour (see Figure 1B), segment, and reconstruct (see Figure 1C) neurons within a neural circuit. 


\section{References}

[1] Leighton SB (1981). SEM images of block faces, cut by a miniature microtome within the SEM - a technical note. Scan Electron Microsc. 1981;(Pt 2):73-6.

[2] Denk W, Horstmann H. (2004). Serial block-face scanning electron microscopy to reconstruct three-dimensional tissue nanostructure. PLoS Biol. 2/e329:1900-1909; 329.

[3] Briggman KL, Denk W (2006) Towards neural circuit reconstruction with volume electron microscopy techniques. Curr Opin Neurobiol 16:562-570.

[4] This work is supported through a grant to ER-M from the NIH NS 39405-07
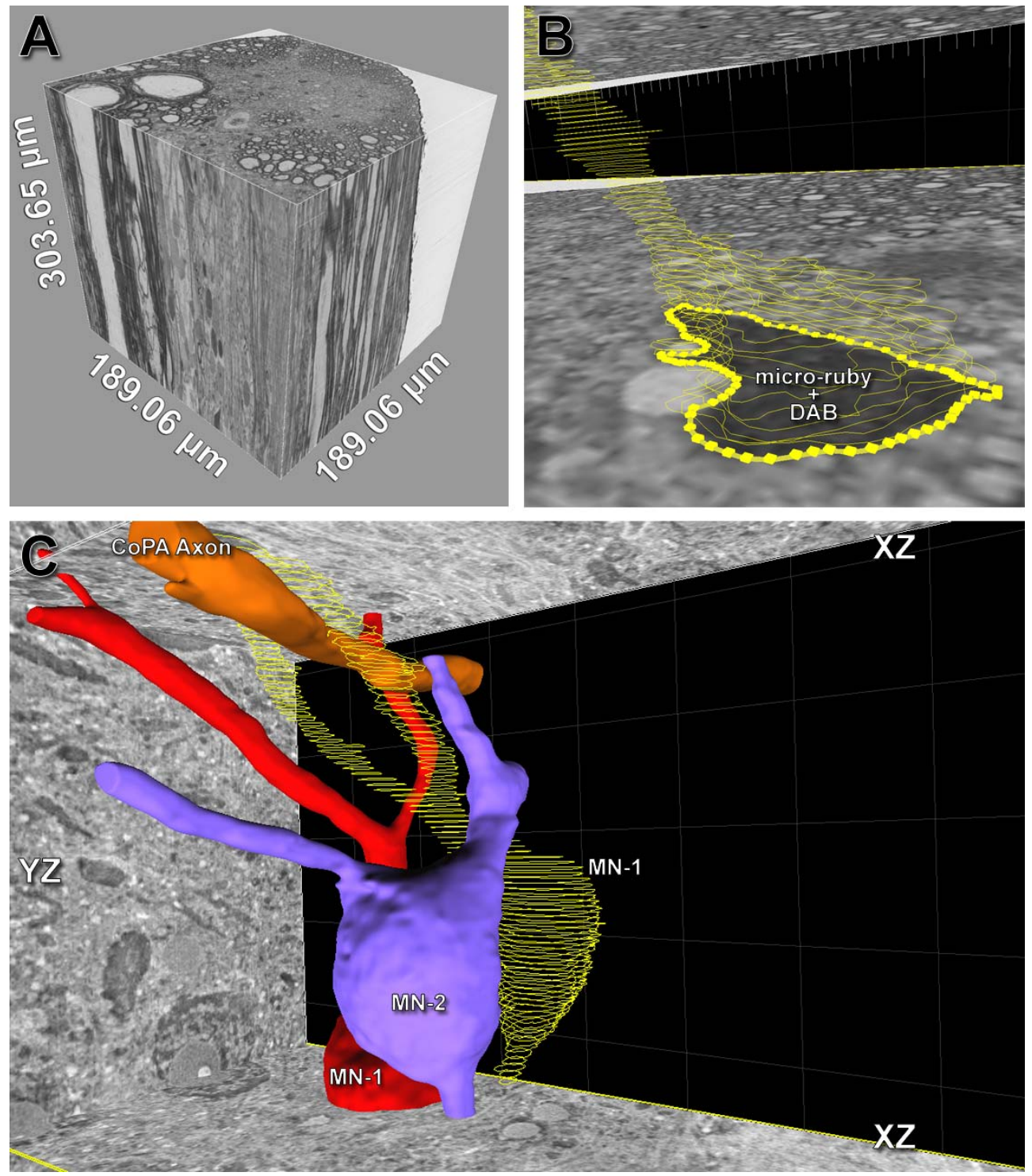

Figure 1. Image stack composed composed of 6,000 images. Note the excellent registration between all images within the image stack (A). The 3,000 MW micro-ruby labeling and visualization with 3'3' diaminobenzadine (DAB) combined with the heavy atom staining provides high contrast at neuronal boundaries, in order to easily contour (B), segment, and reconstruct (C) type-1 and type 2 motor neurons (MN-1) and the axon of a commissarial primary ascending interneuron (CoPA Axon) 